\title{
Evidence of substrate induced charge order quenching, insulator metal transition, and colossal magnetoresistance in polycrystalline $\operatorname{Pr}_{0.58} \mathrm{Ca}_{0.42} \mathrm{MnO}_{3}$ thin films
}

\author{
Vasudha Agarwal, ${ }^{1}$ R. Prasad, ${ }^{1}$ M. P. Singh, ${ }^{2}$ P. K. Siwach, ${ }^{1}$ Amit Srivastava, ${ }^{3}$ \\ P. Fournier, and H. K. Singh ${ }^{1, a)}$ \\ ${ }^{1}$ National Physical Laboratory (CSIR), Dr. K. S. Krishnan Marg, New Delhi 110012, India \\ ${ }^{2}$ Département de Physique and RQMP, Université de Sherbrooke, Sherbrooke, Quebec J1K 2R1, Canada \\ ${ }^{3}$ Department of Physics, Banaras Hindu University, Varanasi 221005, India
}

(Received 6 October 2009; accepted 6 January 2010; published online 5 February 2010)

\begin{abstract}
We report the magnetoelectrical properties of polycrystalline $\operatorname{Pr}_{0.58} \mathrm{Ca}_{0.42} \mathrm{MnO}_{3}$ thin films (thickness $\sim 300 \mathrm{~nm}$ ) deposited on single crystal $\mathrm{LaAlO}_{3}(\mathrm{LAO})$ and $\mathrm{SrTiO}_{3}(\mathrm{STO})$ substrates. The films on LAO show charge ordering $(\mathrm{CO})$ at $T_{\mathrm{CO}} \approx 240 \mathrm{~K}$, with a metamagnetic ground state akin to the cluster glass (CG). In PCMO/STO films the $\mathrm{CO}$ is quenched and enhanced magnetic moment in the CG state suggests stronger ferromagnetic component. The resistivity of the films on LAO and STO differ drastically, the former has temperature dependence typical to the $\mathrm{CO}$ state, while the later show thermal cycling dependent insulator-metal transition (IMT). The large hysteresis in the temperature dependent resistivity provides the evidence of cluster coexistence. The films on STO also exhibit colossal magnetoresistance $(\mathrm{CMR} \sim 91 \%)$ at moderate magnetic field $(\sim 10 \mathrm{kOe})$. The CO quenching, IMT, and CMR are explained in terms of the substrate induced magnetoelectrical phase coexistence. () 2010 American Institute of Physics. [doi:10.1063/1.3302455]
\end{abstract}

Charge-orbital ordering ( $\mathrm{COO})$ and colossal magnetoresistance $(\mathrm{CMR})$ phenomena have been studied extensively in doped rare earth manganites. ${ }^{1-3} \mathrm{Pr}_{1-x} \mathrm{Ca}_{x} \mathrm{MnO}_{3}$ has been recognized as a prototype system in which the competition between charge, spin, and lattice degrees of freedom leads to COO having CE-type antiferromagnetic (AFM) spin order that coexists with a ferromagnetic (FM) phase at low temperature. ${ }^{1,4-6}$ At $0.15<x<0.3$, it is a FM insulator (FMI) at low temperatures and no $\mathrm{COO}$ is observed. The ground state changes to robust $\mathrm{COO}$ insulator (COOI) with an AFM spin order at $0.3<x<0.5$. The magnetic fields needed to melt the COOI are reduced significantly as $\mathrm{Ca}^{2+}$ is lowered from $x=0.5$ to $x=0.3 .^{3-7}$ At $x \sim 0.3-0.5$ it exhibits the CMR, which originates from the competition among the charge ordering due to Coulomb interaction of $\mathrm{Mn}^{3+}$ and $\mathrm{Mn}^{4+}$ ions, magnetic ordering due to superexchange (SE) and doubleexchange (DE) interactions, and orbital ordering of $e_{g}$ electrons of $\mathrm{Mn}^{3+}$ ions in magnetic fields. ${ }^{3-6}$

Perturbations such as electric current, electromagnetic radiation, etc. can also destabilize the COOI state. ${ }^{4-6}$ The COOI is also sensitive to crystallite size and can be suppressed appreciably on downsizing the material to few nanometers. ${ }^{7-10}$ However, in nanomanganites the electrical transport is dominated by the grainboundary (GB) contribution. ${ }^{7-10}$ One of the many clues that can lead to a sound understanding of the highly competitive and correlated physics of manganites is the presence of inhomogeneities and quenched disorder (QD). ${ }^{1,2}$ Disorder is known to weaken the COOI state and presence of QD can have strong disruptive effect. ${ }^{1,2}$ In partially oriented polycrystalline thin films having sufficiently large grain size, the GB contribution is reduced considerably and defects such as oxygen and cationic vacancies can modify the ground state of the system.

${ }^{a)}$ Electronic mail: hks65@mail.nplindia.org.
Oxygen vacancies in particular can introduce spin canting in the background of competing AFM-SE and FM-DE that may evolve into a spin glass (SG) or cluster glass (CG) with a metallic flavor at lower temperatures. The charge order quenching and insulator-metal transition (IMT) with concomitant CMR are observed in compressively strained thin films ${ }^{11}$ and in single crystals under large hydrostatic pressure. ${ }^{12}$ In this letter we have shown that substrate induced oxygen vacancies can cause charge order quenching, promote FMM phase, and produce CMR effect in polycrystalline $\mathrm{Pr}_{0.58} \mathrm{Ca}_{0.42} \mathrm{MnO}_{3}$ (PCMO) thin films.

Polycrystalline PCMO films of thickness $\sim 300 \mathrm{~nm}$ were prepared by ultrasonic nebulized spray pyrolysis ${ }^{13}$ on single crystal $\mathrm{LaAlO}_{3}$ [LAO; (100), $\left.a_{\mathrm{c}}=3.798 \AA\right]$ and $\mathrm{SrTiO}_{3}\left[\mathrm{STO} ;(100), a_{\mathrm{c}}=3.905 \AA\right]$ substrates. All the films were deposited from aqueous solution containing stoichiometric amounts (Pr: $\mathrm{Ca}: \mathrm{Mn}=0.58: 0.42: 1)$ of $\mathrm{Pr}, \mathrm{Ca}$, and $\mathrm{Mn}$ nitrates at substrate temperature $T_{\mathrm{S}} \sim 200{ }^{\circ} \mathrm{C}$ and then annealed in air at $T_{\mathrm{A}} \sim 1000{ }^{\circ} \mathrm{C}$ for $12 \mathrm{~h}$. The structural and surface characterization was done by $\mathrm{x}$-ray diffraction (XRD) and scanning electron microscopy, respectively. The cationic composition was studied by energy dispersive spectroscopy. The magnetization was measured by a commercial SQUID magnetometer (Quantum Design). The electrical resistivity and magnetoresistance (MR) were measured by the four probe method in the temperature range $4.2-350 \mathrm{~K}$ and 77-300 K, respectively.

XRD shows that both films are single phase polycrystalline and have orthorhombic structure. Dominant maxima along (hk0) show the partial orientation in these films. The surface morphology shows that these films mostly consist of grains of size $\sim 300-400 \mathrm{~nm}$. The lattice parameters of the films on different substrates do not show any appreciable difference.

The temperature dependent zero field cooled (ZFC) and field cooled (FC) magnetization $(M-T)$ of both the PCMO 


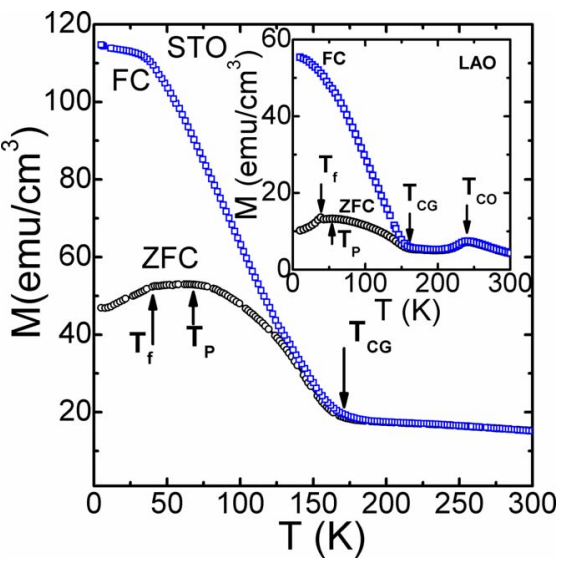

FIG. 1. (Color online) Temperature dependent ZFC and FC magnetization measured at $H=500$ Oe. The data of the film on LAO is plotted in the inset, while the main panel shows the same of the film on STO.

films measured at $H=500$ Oe is shown in Fig. 1. The films on LAO (inset of Fig. 1) show the CO transition at $T^{\mathrm{M}}{ }_{\mathrm{CO}}$ $\approx 240 \mathrm{~K}$ as evidenced by the peak in the $M-T$ curve. This agrees well with the values reported for single crystals, bulk polycrystals, and nanowires. ${ }^{3-9}$ The $M-T$ curve rises around $T_{\mathrm{CG}} \approx 150 \mathrm{~K}$, and a cusplike feature is seen at $T_{\mathrm{P}}=60 \mathrm{~K}$. Below this cusp, the ZFC $M-T$ curve shows a sharp drop at $T_{\mathrm{f}} \approx 42 \mathrm{~K}$. In addition, the ZFC-FC curves show a strong divergence that begins in the vicinity of $T_{\mathrm{CG}}$. In Fig. 1 (main panel), the peak corresponding to $T^{\mathrm{M}}{ }_{\mathrm{CO}}$ is not seen in the $M-T$ data of films on STO. This suggests a quenched CO in this film. Further, the rise in magnetization occurs at $T_{\mathrm{CG}}$ $\approx 180 \mathrm{~K}$ and is considerably sharper than the same observed in the films on LAO. The cusplike feature is also shifted to slightly higher temperature, $T_{\mathrm{P}} \approx 64 \mathrm{~K}$ but the low temperature drop again occurs at $T_{\mathrm{f}} \approx 42 \mathrm{~K}$. The rise in $M$ - $T$ curve at $T_{\mathrm{CG}}$ is a signature of the growth of FM correlations and the higher magnetic moment of the film on STO corresponds to larger FM fraction. Generally, ZFC and FC divergence, the cusplike behavior and the drop in ZFC magnetization at $T_{\mathrm{f}}$ are the typical signatures of metamagnetic systems such as SG/CG ${ }^{14}$ However, typical SG systems are distinguished ${ }^{14}$ by following: (i) the difference between the ZFC and FC magnetization is not as large as in the present case, (ii) the divergence starts at $T \approx T_{\mathrm{f}}$, and (iii) the FC magnetization remains temperature independent at $T<T_{\mathrm{f}}$. Thus the occurrence of a true SG system in the present case is ruled out and we believe that the magnetic order is spin CG type, which consists of FM ordering in an AFM matrix. Due to this reason we have denoted the temperature corresponding to the rise in $M-T$ curves by $T_{\mathrm{CG}}$. The increase in magnetization is a clear evidence of the growth of FM correlations at $T$ $<T_{\mathrm{CO}}$. The sharp drop at $T_{\mathrm{f}}$ is a generic feature of the metastable CG state and may be taken as the temperature below that the clusters freeze. The $M-H$ data (Fig. 2) of these films also support the above observations. The film on LAO shows coercivity $H_{\mathrm{C}}=440$ Oe and the magnetization does not saturate up to magnetic field $H=20 \mathrm{kOe}$. The absence of magnetization saturation reconfirms the coexistence of an AFM and FM phases. The film on STO exhibits a stronger $M-H$ loop with coercivity $H_{\mathrm{C}} \approx 1510$ Oe and saturation magnetization $M_{\mathrm{S}} \approx 480 \mathrm{emu} / \mathrm{cm}^{3}$ around $H \approx 12 \mathrm{kOe}$.

The temperature dependent resistivity $(\rho-T)$ of all PCMO films is given in Fig. 3. The films on LAO substrates

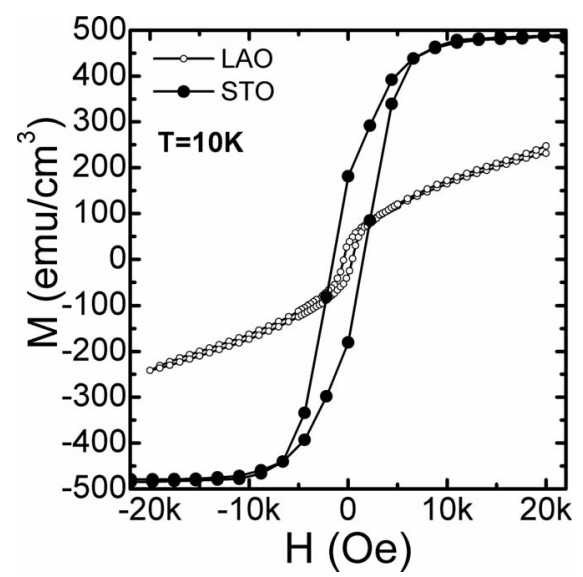

FIG. 2. $M-H$ loop of PCMO films taken at $T=10 \mathrm{~K}$

shows a charge ordering transition at $T^{\rho}{ }_{\mathrm{CO}} \approx 230 \mathrm{~K}$ (shown in the inset of Fig. 3), which agrees well with the CO transition in the $M-T$ data $\left(T^{\mathrm{M}}{ }_{\mathrm{CO}} \approx 240 \mathrm{~K}\right)$. The small difference in the two $T_{\mathrm{CO}}$ 's and the fact that $T^{\rho}{ }_{\mathrm{CO}}<T^{\mathrm{M}}{ }_{\mathrm{CO}}$ could be due to the polycrystalline nature of the films. At $T<T^{\rho}{ }_{\mathrm{CO}}$ resistivity increases further and we could not measure it at $T$ $<70 \mathrm{~K}$ because of the voltage-limit of the current source used. The $\rho-T$ data shows that the film on LAO consists mainly of insulating clusters, which in view of the magnetic properties, could be $\mathrm{PM}\left(T>T_{\mathrm{CO}}\right)$, AFM $\left(T<T_{\mathrm{CO}}\right)$ and $\mathrm{FM}$ $\left(T<T_{\mathrm{CG}}\right)$. However, in view of the intrinsic phase separation tendency of manganites, two or more magnetic phases could coexist. ${ }^{2,3}$ The $\rho-T$ of the films on STO (main panel of Fig. 3) shows some interesting features: (i) the resistivity of this film is considerably smaller than the films on LAO, (ii) the $\mathrm{CO}$ transition is absent, (iii) an IMT is observed at $T<T_{\mathrm{CG}}$, and (iv) the $\rho-T$ curves exhibit a strong hysteresis resulting in large difference in the IMT temperatures, which are $T_{\mathrm{IM}}$ $\approx 108$ and $148 \mathrm{~K}$, respectively, in the cooling and heating cycles. These features provide clear evidence of strong metallic fraction. The large hysteresis in the $\rho-T$ curves is also suggestive of the electronically phase separated state consisting of insulating and metallic clusters. In view of the magnetic properties elucidated earlier, we believe that the films on STO comprise of PMI at $T>T_{\mathrm{CG}}$, and FMM, AFM/COI, and FMI at $T<T_{\mathrm{CG}}$. In the CG state, the order parameters of the AFM/COOI and FMM are randomly oriented. We would like to mention here that IMT has not been observed earlier

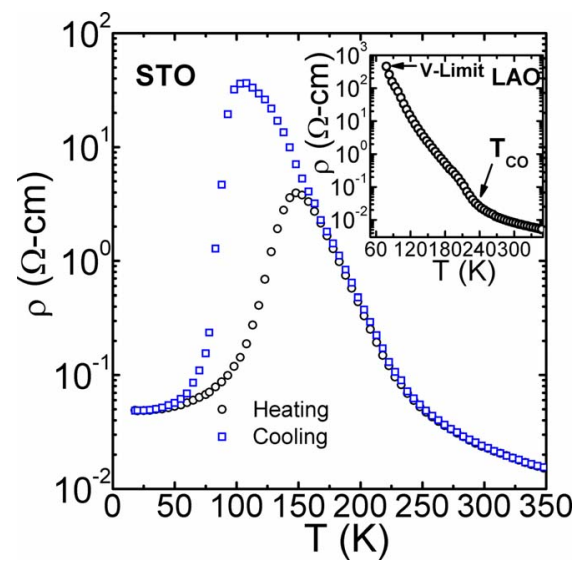

FIG. 3. (Color online) Temperature dependent resistivity of PCMO films deposited on LAO substrate (inset) and STO (main panel). 


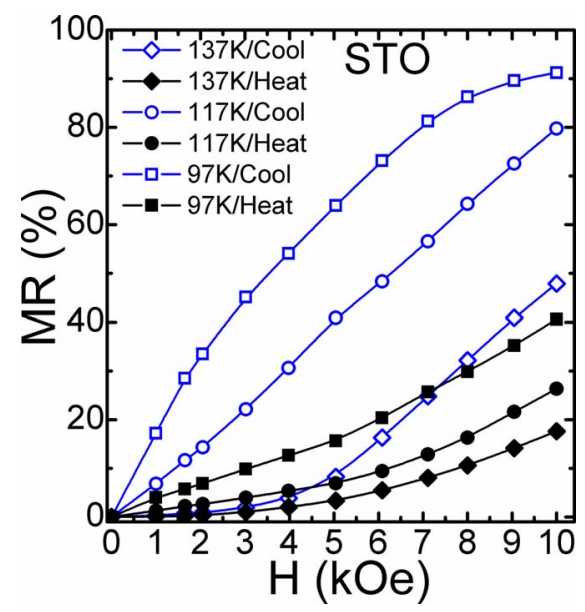

FIG. 4. (Color online) Magnetic field dependence of the isothermal magnetoresistance of the PCMO film on STO measured during heating and cooling.

in PCMO in absence of an external perturbation such as magnetic field, etc.

The films on LAO do not show MR $\{\mathrm{MR}=100 x[\rho(H$ $=0)-\rho(H)] / \rho(H=0)\}$ in magnetic fields up to $10 \mathrm{kOe}$ in the temperature range $300-77 \mathrm{~K}$. In contrast, the films on STO showed large MR which is strongly dependent on the thermal cycling (Fig. 4). During the cooling cycle $\left(T_{\mathrm{IM}}\right.$ $\approx 108 \mathrm{~K}$ ), the maximum $\mathrm{MR} \approx 34 \%$ and $91 \%$ at $H=2$ and $10 \mathrm{kOe}$, respectively, is observed at $97 \mathrm{~K}$. The MR is considerably reduced during the heating cycle $\left(T_{\mathrm{IM}} \approx 148 \mathrm{~K}\right)$ and the highest $\mathrm{MR} \approx 40 \%$ at $H=10 \mathrm{kOe}$ is observed at $97 \mathrm{~K}$.

The destabilization of COOI in films on STO is also in contrast to the nearly similar epitaxial films on STO in which it is strongly enhanced. ${ }^{15}$ The present results can be understood in terms of the substrate induced effects. The cubic lattice constant corresponding to the orthorhombic unit cell of PCMO $\left(a_{\mathrm{c}} \approx 3.82 \AA\right)$, is very close to that of the LAO (100) but smaller than that of the STO (100). Consequently, during the growth, the energy of the PCMO-STO interface is expected to be much larger than that of the PCMO-LAO. The energy minimization to achieve stable structural/ microstructural configuration results in the creation of defects such as oxygen vacancies, stacking faults, dislocations, etc. and the films having larger interfacial energy are expected to have higher density of such defects. Since all the films are annealed in air, the role of oxygen vacancies is expected to be dominant. To evaluate the role of oxygen vacancies in the quenching of the COOI state we annealed some PCMO films in flowing oxygen. The magnetization and resistivity measurements on these films (results not shown) reveal that the properties of the film on LAO remain nearly unaffected. In contrast, the $M-T$ of the film on STO shows a $\mathrm{CO}$ transition at $T^{\mathrm{M}}{ }_{\mathrm{CO}} \approx 235 \mathrm{~K}$ with simultaneous disappearance of the IMT and the CMR. The $\rho-T$ data of the oxygenated film on STO shows only a plateau-like feature in the vicinity of the IMT of the air annealed film. This shows that the oxygen vacancies are the major source of destabilization of the COOI state. Oxygen vacancies reduce the effective hole concentration and an inhomogeneous distribution of these could act as QD. We would like to mention that $\mathrm{CO}$ melting induced by oxygen vacancies has been reported recently in $\mathrm{CaMnO}_{3}$ single crystals also. ${ }^{16}$ At sufficiently small grain size $(\leq 40 \mathrm{~nm})$ the $\mathrm{CO}$ is destabilized and CG like state appears also in bulk PCMO samples but IMT and CMR are not observed. ${ }^{10}$ This could, possibly be due to the dominance of GB disorder over the oxygen vacancy induced QD. The substrate induced partially oriented growth in polycrystalline thin films results in better crystallographic and microstructural order, which suppresses the GB disorder considerably and makes the QD dominant. Although, it will be interesting to observe these properties in bulk PCMO, our results suggest that substrate-induced vacancies play a crucial role. This may hinder to replicate the similar nature of oxygen vacancies in the bulk polycrystalline samples and hence a similar behavior. In this regard a substrate like STO seems essential.

The strong hysteresis in the resistivity and large difference in MR could be understood in terms of the CG concept. During the cooling cycle the spin CG mimics a liquid like behavior. Due to this carrier scattering by disordered spins is strong resulting in a large resistivity and the lower $T_{\mathrm{IM}}$. The carrier scattering is strongly reduced due to induced spin order when magnetic field is applied. This causes a sharp drop in the resistivity and hence the huge MR during the cooling cycle. When cooled to $T<T_{\mathrm{f}}$ the $\mathrm{CG}$ is frozen and the carrier scattering is suppressed considerably. This results in lower resistivity and higher $T_{\mathrm{IM}}$ during the heating cycle. When the CG is frozen much larger magnetic fields are required to align the magnetic moments and consequently MR is reduced considerably during the heating cycle.

In conclusion, we have studied the magnetoelectrical properties of polycrystalline $\mathrm{Pr}_{0.58} \mathrm{Ca}_{0.42} \mathrm{MnO}_{3}$ thin films deposited on LAO and STO substrates. We have shown that the substrate induced oxygen vacancies can destabilize the $\mathrm{CO}$, induce hysteretic IMT and cause CMR at moderate magnetic field.

V.A. thanks CSIR/NPL for research internship. P.F. and M.P.S. acknowledge the financial support of CFI, CIFAR, NSERC (Canada). and FQRNT (Quebec).

${ }^{1}$ Y. Tokura, Rep. Prog. Phys. 69, 797 (2006).

${ }^{2}$ E. Dagotto, New J. Phys. 7, 67 (2005).

${ }^{3}$ C. N. R. Rao, A. Arulraj, A. K. Cheetham, and B. Raveau, J. Phys.: Condens. Matter 12, R83 (2000).

${ }^{4}$ Y. Tomioka, A. Asamitsu, H. Kuwahara, Y. Moritomo, and Y. Tokura, Phys. Rev. B 53, R1689 (1996).

${ }^{5}$ A. Asamitsu, Y. Tomioka, H. Kuwahara, and Y. Tokura, Nature (London) 388, 50 (1997).

${ }^{6}$ K. Miyano, T. Tanaka, Y. Tomioka, and Y. Tokura, Phys. Rev. Lett. 78, 4257 (1997)

${ }^{7}$ C. L. Lu, S. Dong, K. F. Wang, F. Gao, P. L. Li, L. Y. Lv, and J.-M. Liu, Appl. Phys. Lett. 91, 032502 (2007).

${ }^{8}$ S. S. Rao, K. N. Anuradha, S. Sarangi, and S. V. Bhata, Appl. Phys. Lett. 87, 182503 (2005).

${ }^{9}$ A. Biswas and I. Das, Appl. Phys. Lett. 91, 013107 (2007).

${ }^{10}$ T. Zhang and M. Dressel, Phys. Rev. B 80, 014435 (2009).

${ }^{11}$ W. Prellier, A. Biswas, M. Rajeswari, T. Venkatesan, and R. L. Greene, Appl. Phys. Lett. 75, 397 (1999).

${ }^{12}$ C. Cui and T. A. Tyson, Phys. Rev. B 70, 094409 (2004)

${ }^{13}$ P. K. Siwach, V. P. S. Awana, H. Kishan, R. Prasad, H. K. Singh, S. Balamurugan, E. Takayama-Muromachi, and O. N. Srivastava, J. Appl Phys. 101, 073912 (2007).

${ }^{14}$ J. A. Mydosh, Spin Glasses: An Experimental Introduction, 2nd ed. (Taylor \& Francis, London, 1993).

${ }^{15}$ Z. Q. Yang, Y. Q. Zhang, J. Aarts, M.-Y. Wu, and H. W. Zandbergen, Appl. Phys. Lett. 88, 072507 (2006).

${ }^{16}$ N. N. Loshkareva, N. V. Mushnikov, A. V. Korolyov, E. A. Neifeld, and A. M. Balbashov, Phys. Rev. B 77, 052406 (2008). 